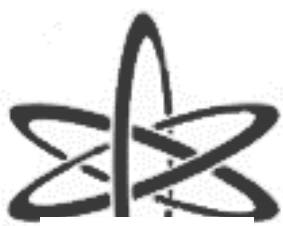

BJRS
BRAZILIAN JOURNAL

$\mathrm{OF}$

RADIATION SCIENCES

05-01 (2017) 01-16

\title{
Avaliação das exposições dos envolvidos em procedimentos intervencionistas usando método Monte Carlo
}

\author{
W. S. Santos ${ }^{\mathrm{a}, \mathrm{b}}$; L. P. Neves ${ }^{\mathrm{b}}$; A. P. Perini ${ }^{\mathrm{b}}$; W. Belinato ${ }^{\mathrm{c}}$; \\ A. F. Maiad; L. V. E. Caldas ${ }^{\mathrm{a}}$. \\ ${ }^{a}$ Instituto de Pesquisas Energéticas e Nucleares (IPEN-SP),São Paulo, SP, Brasil \\ ${ }^{b}$ Instituto de Física, Universidade Federal de Uberlândia (UFU),Uberlândia, MG, Brasil \\ 'Instituto Federal da Bahia (IFBA), Vitória da Conquista, BA, Brasil \\ ${ }^{d}$ Universidade Federal de Sergipe (UFS), São Cristovão, SE, Brasil \\ williathan@yahoo.com.br
}

\section{RESUMO}

Neste estudo apresenta-se um modelo computacional de exposição de um paciente, médico cardiologista e de uma enfermeira em um cenário típico de procedimentos intervencionistas cardíacos. Neste caso, foi calculado um conjunto de coeficientes de conversão (CC) para dose efetiva (E) em termos do produto kerma-área (PKA) para os indivíduos envolvidos, utilizando sete espectros energéticos diferentes e oito projeções de feixe. Também foi calculado o CC para dose de entrada na pele (DEP) do paciente normalizado pelo PKA. Todos os indivíduos foram representados por simuladores antropomórficos computacionais incorporados em um código de transporte de radiação baseado em simulação de Monte Carlo.

Palavras - chave: Dose de radiação, radiologia intervencionista, simulação de Monte Carlo.

\section{ABSTRACT}

This study presents a computational model of exposure of a patient, a cardiologist and a nurse in a typical scenario of cardiac interventional procedures. In this case, a set of conversion coefficients (CC) for effective dose (E) in terms of the kerma-area product (KAP) was calculated for the individuals involved. For this, seven different energy spectra and eight beam projections were used. We also calculated the CC for the skin entrance dose (ESD) of the patient normalized by KAP. All individuals were represented by virtual anthropomorphic phantoms inserted in a radiation transport code based on Monte Carlo simulation. 


\section{INTRODUÇÃO}

Nos últimos anos, houve um crescente avanço no número de procedimentos de radiologia intervencionista (RI), que resultaram em um aumento na frequência de procedimentos de diagnóstico e de terapia, utilizando raios X (Faulkner, 2008). O uso médico da radiação ionizante oferece grandes benefícios para os pacientes. Entretanto, há uma crescente preocupação com o monitoramento das doses de radiação recebidas por pacientes e profissionais médicos submetidos a procedimentos de RI guiado por fluoroscopia. Em RI, o médico utiliza imagens contínuas de raios $\mathrm{X}$ para guiar os instrumentos médicos através dos vasos do paciente e por outras vias de acesso (Wagner et al, 2000).

Uma vez que estes procedimentos geralmente requerem menor tempo de recuperação e resultam em menos complicações pós-operatórias, tornaram-se cada vez mais comuns. Na maioria das vezes, estes procedimentos dispensam intervenções cirúrgicas complexas (Miller, 2008). Apesar destas vantagens em relação às técnicas invasivas, existem vários riscos associados aos procedimentos intervencionistas como resultado do uso da radiação ionizante.

Diversos casos de danos por radiação em pacientes e médicos foram relatados devido a exposições elevadas de raios $\mathrm{X}$ durante procedimentos de fluoroscopia (Khodadadegan et al, 2011). Por serem exames complexos, os procedimentos cardíacos de angiografia e angioplastia coronária em RI são longos e, muitas vezes, com a obtenção de várias imagens, o que explica os altos valores de dose associados à prática. A ocorrência de efeitos determinísticos, especialmente na pele do paciente, é frequente após a cirurgia e tem sido um assunto de grande preocupação na comunidade científica (Faulkner, 2008).

Atualmente, apenas a dosimetria retrospectiva é possível. Neste caso, os pacientes são informados se eles devem procurar tratamento médico por lesões induzidas por radiação. Felizmente, os avanços tecnológicos modernos levaram à oportunidade de avaliação de dose na pele em tempo real. Além disso, a estimativa do risco para a saúde devido a efeitos estocásticos da radiação, especialmente para pessoas mais jovens, também é objeto de estudos mais aprofundados (ICRP, 2013). 
As exposições ocupacionais em procedimentos de RI são as mais altas na área da medicina que utiliza radiação X (Falkner e Vañó, 2001). Em RI, na maioria das vezes é necessário que os profissionais intervencionistas se posicionem próximos do centro do campo de radiação e do tubo de raios $\mathrm{X}$, utilizando um grande número de imagens estáticas e dinâmicas, levando a um tempo alto de exposição destes indivíduos. Assim, a exposição prolongada de pacientes e profissionais médicos aos raios $\mathrm{X}$ tem que ser investigada. Portanto, é importante desenvolver novos métodos que possibilitem estimar as doses equivalentes nos órgãos destas pessoas com boa precisão, que na maioria das vezes é um processo complexo e impossível de ser realizado experimentalmente. Neste caso, a estimativa das grandezas dosimétricas tem sido feita utilizando-se códigos de transporte de radiação fundamentado em simulação Monte Carlo e simuladores antropomórficos para representar os indivíduos expostos (Santos et al, 2014).

O objetivo deste trabalho foi criar um modelo computacional de exposição que permitisse a avaliação das exposições de um paciente, um médico cardiologista e de uma enfermeira devido a procedimentos de angiografia e angioplastia coronária em RI. As projeções simuladas são as mais comuns e se aplicam a muitos procedimentos de RI cardíacas e não exclusivamente a coronariografia e angioplastia.

\section{MATERIAIS E MÉTODOS}

A avaliação dosimétrica foi feita por meio da determinação dos coeficientes de conversão (CC) para a dose efetiva (E), para todos os indivíduos, e para a dose de entrada na pele (DEP) do paciente, normalizados pelo produto kerma-área (PKA). Estes CCs foram calculados para uma amplo intervalo de espectro energético, que abrange grande parte dos procedimentos cardíacos realizados em RI.

O código de transporte de radiação MCNPX 2.7.0 (Pelowitz, 2011) foi usado para simular a deposição energética de fótons e elétrons nos órgãos e tecidos dos simuladores antropomórficos adultos, masculino (MASH) e feminino (FASH). Estes simuladores foram desenvolvidos pelo o grupo de dosimetria computacional do Departamento de Energia Nuclear da Universidade Federal de Pernambuco (DEN/UFPE), cujas características atendem às recomendações dos dados 
anatômicos e fisiológicos do homem e da mulher de referência da ICRP 89 (Cassola et al, 2010). O simulador antropomórfico virtual MASH foi utilizado para representar o paciente e o médico cardiologista. A enfermeira foi representada pelo simulador virtual FASH. Os simuladores foram incorporados ao código de transporte de radiação MCNPX 2.7.0. Esse código descreve o transporte de um conjunto de partículas tais como fótons, elétrons, nêutrons, nucleons, íons leves, partículas beta, partículas alfa, prótons, etc. Além dessas partículas, o código também transporta partículas secundárias produzidas. No código MCNPX, foi possível simular o transporte de fótons incluindo os principais processos de interação como absorção fotoeléctrica com a possibilidade de emissão fluorescente e elétron Auger, espalahamento coerente e incoerente. Durante o transporte de fótons e elétrons, foi utilizada a biblioteca de seção de choque ENDF/B-VI8 do código para descrever os possíveis efeitos no meio. Além disso, neste estudo, os valores de energia de corte para fótons e elétrons, tipo de interação, seção de choque e técnica de redução de variância foram mantidas como valores padrões do código.

Para evitar problemas de alocação de memória, as matrizes originais dos simuladores compostas de voxels de 1,2 $\mathrm{mm}$ de comprimento foram redimensionadas para 2,4 mm cada um, sendo os dados antropométricos dos simuladores preservados. Para avaliar a influência destas modificações, foram feitas simulações com o simulador antropomórfico computacional com voxel cúbicos de 1,2 mm de aresta e verificou-se que não houve alterações importantes nos resultdos.

Todos os equipamentos e objetos simuladores foram inseridos em uma sala com dimensões de 6,5 $\mathrm{m}$ de comprimento, 3,0 $\mathrm{m}$ de largura e 2,65 $\mathrm{m}$ de altura preenchidas com ar atmosférico ( $\left.\rho=0,001205 \mathrm{~g} / \mathrm{cm}^{3}\right)$ composto por C $(0,0124 \%), \mathrm{N}(75,53 \%)$, O $(23,18 \%)$ e $\operatorname{Ar}(1,28 \%)$ e com paredes de concreto $\left(\rho=3,2 \mathrm{~g} / \mathrm{cm}^{3}\right)$ de $22 \mathrm{~cm}$ de espessura.

Também foi modelada uma mesa cirúrgica de fibra de carbono $\left(\rho=1,25 \mathrm{~g} / \mathrm{cm}^{3}\right)$ composta por H (5,7441\%), C (77,4591\%) e O (16,7968\%), com base metálica ( $\left.\rho=7,87 \mathrm{~g} / \mathrm{cm}^{3}\right)$ composta de Fe (99,5\%) e Mn (0,5\%) para receber o paciente que é colocado em decúbito dorsal sobre um colchão de espuma ( $\left.\rho=1,0 \mathrm{~g} / \mathrm{cm}^{3}\right)$. A mesa tem uma largura de $66 \mathrm{~cm}$, espessura de $15 \mathrm{~cm}$ e comprimento de $185 \mathrm{~cm}$ e está posicionada a uma altura de $90 \mathrm{~cm}$ em relação ao piso da sala. A Figura 1 mostra o modelo computacional de exposição do paciente, médico cardiologista, enfermeira e grande parte dos equipamentos típicos uma sala de RI. 
Figura 1. Modelo computacional de exposição: (1) médico cardiologista, (2) paciente, (3) enfermeira, (4) mesa cirúrgica, (5) mesa de instrumentação, (6) tubo de raios $X$, (7) intensificador de imagem, (8) medidor de PKA, (9) medidor de DEP, (10) monitores de vídeo, (11) avental de chumbo, (12) protetor de tireoide, (13) óculos plumbíferos, (14) cortina de chumbo, (15) barreira de proteção suspensa de vidro plumbífero.



Na prática clínica, o cardiologista e a enfermeira utilizam equipamentos de proteção individual que geralmente são confeccionados com $0,5 \mathrm{~mm}$ de espessura de chumbo $(\mathrm{Pb})$. Dentre os equipamentos mais importantes estão o avental e protetor de tireoide composto de $\mathrm{Pb}$ cuja densidade é de $\rho=11,35 \mathrm{~g} / \mathrm{cm}^{3}$, e protetor de olhos, composto de vidro plumbífero $(\rho=6,22$ $\mathrm{g} / \mathrm{cm}^{3}$ ). Os aventais modelados possuem uma parte frontal e outra traseira com formato de elipse. Este modelo tem 1,20 m de comprimento e $55 \mathrm{~cm}$ de largura, que se estende do nível do tórax até o nível dos joelhos, deixando as partes laterais dos ombros e braços dos profissionais expostos.

Também foram inseridos os protetores de olhos, que têm formato cilíndrico $(\mathrm{r}=3 \mathrm{~cm})$ e protetor de tireoide com formato de anel $(r=7,95 \mathrm{~cm})$ cobrindo toda a região do pescoço dos profissionais. Além destes equipamentos de proteção, também foram modelados a cortina de chumbo e os protetores suspensos de vidro com espessura equivalente em chumbo de 0,5 mm. Estes equipamentos são frequentemente utilizados para a proteção dos profissionais médicos que são expostos à radiação espalhada do paciente e da mesa cirúrgica.

O tubo de raios $\mathrm{X}$ foi representado por uma fonte pontual de fótons que emite um feixe cônico de radiação na região do tórax do paciente. Foi modelada uma câmara de ionização com dimensões 
de $5 \mathrm{~cm}$ x $5 \mathrm{~cm}$ e espessura de $1 \mathrm{~cm}$, preenchida com ar atmosférico para calcular o PKA. Esta câmara de ionização foi posicionada na saída do tubo de raios X. O PKA foi calculado por meio do produto do kerma no ar ( $\left.\mathrm{K}_{\mathrm{ar}}\right)$ (obtido pelo tally F6) estimado no volume de ar da câmara de ionização pela sua área irradiada.

Com o objetivo de avaliar os possíveis efeitos determinísticos na pele do paciente, foi modelado um objeto que simula uma câmara de ionização com dimensões do campo irradiado $(10 \mathrm{~cm} \mathrm{x}$ $10 \mathrm{~cm}$ ) para o cálculo dos CCs para dose de entrada na pele (DEP) normalizados pelo PKA. Em todas as projeções, a câmara de ionização foi posicionada próxima à pele do paciente e perpendicular ao eixo do feixe de radiação. A estimativa da DEP em procedimentos de RI foi feita utilizando o tally F6 do MCNPX, que considera o equilíbrio eletrônico. Para todos os indivíduos expostos, foi utilizado o tally modificado (*F8), que é utilizado para estimar a deposição energética nos órgãos e tecidos, que foi posteriormente convertida para dose absorvida.

Os CCs para E e DEP em termos do PKA para os órgãos e os tecidos foram calculados utilizando sete valores de tensão de pico no tubo de 60 a $120 \mathrm{kVp}$ em passos de $10 \mathrm{kVp}$ para oito projeções de feixe listadas na ICRP (ICRP, 2013). A projeção do feixe e a correspondente distância focopele (DFP) simuladas foram: ântero-posterior $(\mathrm{AP} / 55 \mathrm{~cm})$, póstero-anterior (PA/50 cm), oblíquoanterior direito (RAO90\% $/ 40 \mathrm{~cm})$, oblíquo-anterior esquerdo (LAO90\% $/ 40 \mathrm{~cm})$, caudal (CAUD30\%43,5 cm), cranial (CRAN30\%/45 cm) e oblíquo-anterior direito e anterior esquerdo (RAO45\%/40 cm e LAO45\%56,5 cm). Os parâmetros técnicos utilizados nas simulações foram: filtração no tubo de $3,5 \mathrm{mmAl}$, ângulo anódico de $12^{\circ}$, área de campo de radiação de $10 \mathrm{~cm} \mathrm{x}$ $10 \mathrm{~cm}$. Estes dados foram utilizados para simular os espectros de radiação, com o código SRS78 (Cranley et al, 1997).

Com o intuito de reduzir as incertezas estatísticas (Tipo A) associadas à energia absorvida nos órgãos, tecidos e outras estruturas, foram utilizados em cada cenário um total de $1,0 \times 10^{9}$ histórias de partículas. Estas incertezas foram calculadas utilizando os erros relativos fornecidos pelo código MCNPX. A validação dos resultados foi feita por meio da comparação com resultados obtidos por estudos experimentais publicados na literatura. Todas as simulações foram feitas em um computador de 16 GB de memória RAM com processador Intel Core i7. 
Neste estudo, os resultados dos CCs para dose efetiva e de dose de entrada na pele foram expressos associados com incerteza Tipo A ( $\sigma_{A} \dot{i}$, que foi considerada igual ao desvio padrão. Para cada amostra, o código MCNPX calcula a média de uma grandeza ( $x \dot{i}$ associada a um desvio padrão ( $\sigma_{\dot{x}}$,), que é expresso por meio da seguinte equação:

$$
\sigma_{\dot{x}}=R * \dot{x}
$$

(Eq.1)

onde $\mathrm{R}$ é o erro relativo, que representa a precisão estatística, que é tão pequeno à medida que aumenta o número de histórias simuladas. Conforme o manual do código, quanto menor for R, melhor é a precisão da grandeza estimada. Normalmente, se tem um bom resultado quando é aumentado o número de histórias (Pelowitz, 2011).

\section{RESULTADOS E DISCUSSÕES}

Depois de estimadas as doses equivalentes por PKA para todos os órgãos críticos listados na ICRP (ICRP, 2007), foi possível calcular a razão E/PKA para todos os indivíduos estudados. Os CCs calculados para E e DEP, apresentados neste estudo, são específicos para o modelo de simulador antropomórfico utilizado e para as situações de exposição descritas anteriormente.

\section{Avaliação dos CCs para dose efetiva (E) do paciente}

Embora a ICRP aconselha o uso restrito de dose efetiva para exposições médicas dos pacientes "em radiodiagnóstico, o valor de dose efetiva pode ser utilizado para comparar as doses relativas de diferentes procedimentos diagnósticos e para comparar o uso de tecnologias e procedimentos similares em diferentes hospitais e países, bem como o uso de diferentes tecnologias para o mesmo exame médico, desde que o paciente de referência ou as populações de pacientes sejam semelhantes em relação à idade e ao sexo "(ICRP 2007), tornou-se comum em muitas publicações usar este conceito. Isso se deve principalmente porque essa grandeza pode fornecer uma boa avaliação para o efeito estocástico e, por isso, CCs para esta grandeza (E/PKA) para o 
paciente também foram apresentados neste trabalho. A Figura 2 mostra os principais resultados de E/PKA calculados neste estudo em função da tensão de pico do tubo de raios X e da projeção do feixe para o paciente. Observa-se que os CCs geralmente seguem uma tendência crescente com o aumento da tensão aplicada ao tubo de raios $\mathrm{X}$, que é esperado uma vez que os fótons mais energéticos produzem maiores CCs de dose equivalente, resultando em maiores CCs para a dose efetiva. Os maiores valores de E/PKA foram calculados na projeção CRAN30 e a menor na projeção $\mathrm{LAO}^{\circ} 5^{\circ}$.

Figura 2. Razão E/PKA em função da tensão aplicada ao tubo, calculados para o paciente.

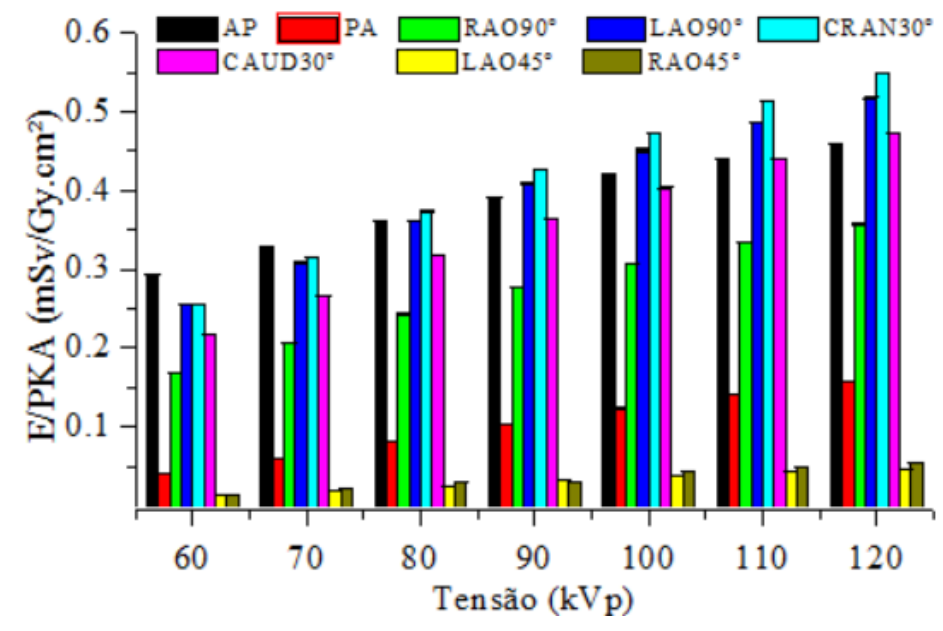

\section{Comparação com valores experimentais e teóricos publicados na literatura}

O valor médio de E/PKA de todos as projeções em nosso estudo para o paciente foi de $(0,25 \pm 0.03) \mathrm{mSv} / \mathrm{Gy} \cdot \mathrm{cm}^{2}$. Este valor é consistente com os dados de medição na literatura como mostrado na Tabela 1. Na mesma tabela, são apresentadas as diferenças percentuais entre o valor de CC obtido neste estudo e os valores da literatura.

Tabela 1. Comparação entre os resultados de E/PKA calculados neste trabalho para o paciente $(0,25 \pm 0.03) \mathrm{mSv} / \mathrm{Gy} \cdot \mathrm{cm}^{2}$ e os descritos na literatura.

\begin{tabular}{ccc}
\hline Referências & $\begin{array}{c}\text { E/PKA } \\
\text { mSv/Gy.cm }\end{array}$ & $\begin{array}{c}\text { Dif. } \\
\text { (\%) }\end{array}$ \\
\hline Betsou et al, 1998 & 0,184 & 26 \\
Struelens et al, 2007 & 0,245 & 2 \\
NCRP, 2009 & 0,260 & -4
\end{tabular}




$\begin{array}{ccc}\text { Park et al, 2008 } & 0.260 & -4 \\ \text { EC, 2008 } & 0,200 & 20 \\ \text { Katritsis et al, 2000 } & 0,182 & 27 \\ \text { Bor et al, 2004 } & 0,240 & 4 \\ \text { Média } & 0,224 \pm 0,03 & 10\end{array}$

Os valores de E/PKA calculadas para o paciente utilizando o simulador MASH foram comparados também com um estudo que utilizou um simulador antropomórfico matemático adulto incorporado no código PCXMC (Tapiovara et al, 1997). A média dos valores E/PKA utilizando o simulador matemático para uma tensão de pico de $100 \mathrm{kVp}$ foi de $0,20 \mathrm{mSv} / \mathrm{Gy} \cdot \mathrm{cm}^{2}$ para o MIRD5/PCXMC (LAO45: 0,26; PA: 0,19; CAUD30: 0,19; RAO90: 0,18). Para estas quatro projeções, o CC médio para a dose efetiva do presente estudo foi de 0,26 mSv/Gy.cm², apresentando um aumento de 23\% em relação ao simulador antropomórfico matemático.

Ao contrário dos resultados do PCXMC, os resultados apresentados neste estudo foram calculados utilizando o paciente sobre uma mesa cirúrgica acompanhado do médico cardiologista e de uma enfermeira. O paciente, representado pelo o simulador antropomórfico MASH, possui uma anatomia mais realística do que os simuladores matemáticos e/ou dos objetos físicos utilizados para representar o paciente em procedimentos de RI. Diferentemente de outros estudos, o simulador antropomórfico MASH foi construído na posição supina incluindo os efeitos gravitacionais na distribuição e acomodação dos órgãos e tecidos no corpo e, por isso, diferenças nos resultados comparados com a literatura foram esperadas e comprovadas. Além destes, foi introduzida na sala de RI uma série de equipamentos típicos empregados nestes procedimentos. Certamente o detalhamento do cenário introduz objetos atenuadores e espalhadores e as diferenças anatômicas e morfológicas entre os simuladores justificam as diferenças entre os estudos.

\section{Avaliação dos CCs para dose de entrada na pele (DEP) do paciente}

Devido à possibilidade de sintomas agudos da radiação na pele, são apresentados resultados da DEP/PKA, que levam em consideração as informações sobre o local e as dimensões de campo. Isto possibilita avaliar a distribuição de dose absorvida na pele irradiada do paciente. A Figura 3 mostra os principais valores da razão DEP/PKA em função da tensão aplicada no tubo e da projeção do feixe de raios $\mathrm{X}$ no paciente. 
Nota-se diferenças entre os valores da DEP/PKA para as projeções avaliadas. Quando o tubo está em baixo da mesa (PA, RAO45 e $\mathrm{LAO}^{\circ} 5^{\circ}$ ), ela atenua o feixe consideravelmente. A principal consequência disso são CCs menores em relação às projeções oblíquas (CRAN30 e CAUD30), lateral (LAO90 ${ }^{\circ}$ e RAO90 ${ }^{\circ}$ e (AP). Nestes casos, o tubo de raios X está acima da mesa e o feixe é projetado diretamente para a pele do paciente, sem a presença de objetos atenuadores.

Figura 3. Razão DEP/PKA calculados para o paciente para as oito projeções de feixe estudadas em função da tensão aplicada no tubo.

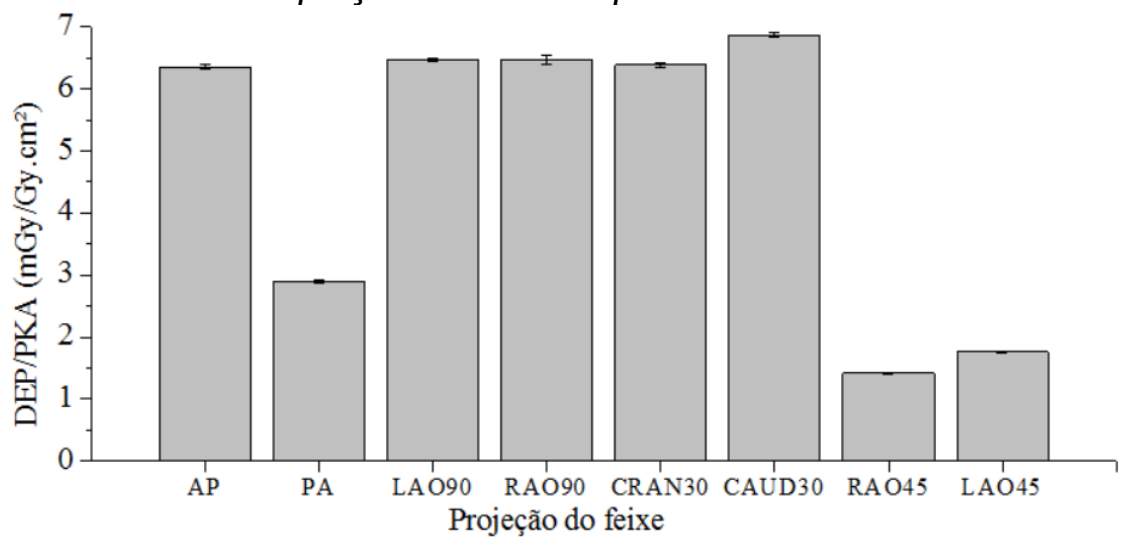

A média da razão DEP/PKA calculada neste estudo foi de $(4,8 \pm 0,1) \mathrm{mGy} / \mathrm{Gy} \cdot \mathrm{cm}^{2}$. Na ausência de uma medição direta, estes CCs podem ser utilizados para determinar a DEP do pacinete. Na Tabela 2 são apresentadas as diferenças entre os resultados deste estudo e os relatados na literatura.

Tabela 2. Comparação entre os resultados de DEP/PKA do paciente obtidos neste estudo (4,8 \pm $0,1) \mathrm{mGy} / \mathrm{Gy} . \mathrm{cm}^{2}$ e os descritos na literatura.

\begin{tabular}{ccc}
\hline Referências & $\begin{array}{c}\text { DEP/PKA } \\
\text { mGy/Gy.cm }\end{array}$ & $\begin{array}{c}\text { Diferença } \\
\text { percentual } \\
\text { (\%) }\end{array}$ \\
\hline Karambatsakidou et al., 2005 & 3,9 & 19 \\
Chida et al., 2006 & 3,8 & 21 \\
Quai et al., 2003 & 4,3 & 10 \\
William et al., 2015 & 4,6 & 4,2 \\
Média & $4,1 \pm 0,3$ & 17 \\
\hline
\end{tabular}


Doses altas na pele são mais prováveis quando o tempo de algum procedimento de fluoroscopia for alto, ou quando o feixe é projetado em uma única direção. Nestes casos, é recomendável monitorar a DEP, para evitar danos na pele do paciente. Com a intenção de evitar os efeitos determinísticos das radiações ionizantes, pode-se empregar a razão DEP/PKA. Este valor pode auxiliar o médico a, por exemplo, modificar o ângulo do feixe quando o valor da DEP alcançar um limite conhecido.

\section{Avaliação dos CCs para dose efetiva (E) do médico cardiologista e da enfermeira}

Os valores de E/PKA calculados para a enfermeira e para o médico cardiologista nas oito projeções estudadas, em função da tensão aplicada ao tubo, estão apresentados na Figura 4.

Figura 4. Razão E/PKA em função da tensão aplicada ao tubo, calculados para o médico cardiologista (A) e a enfermeira (B).

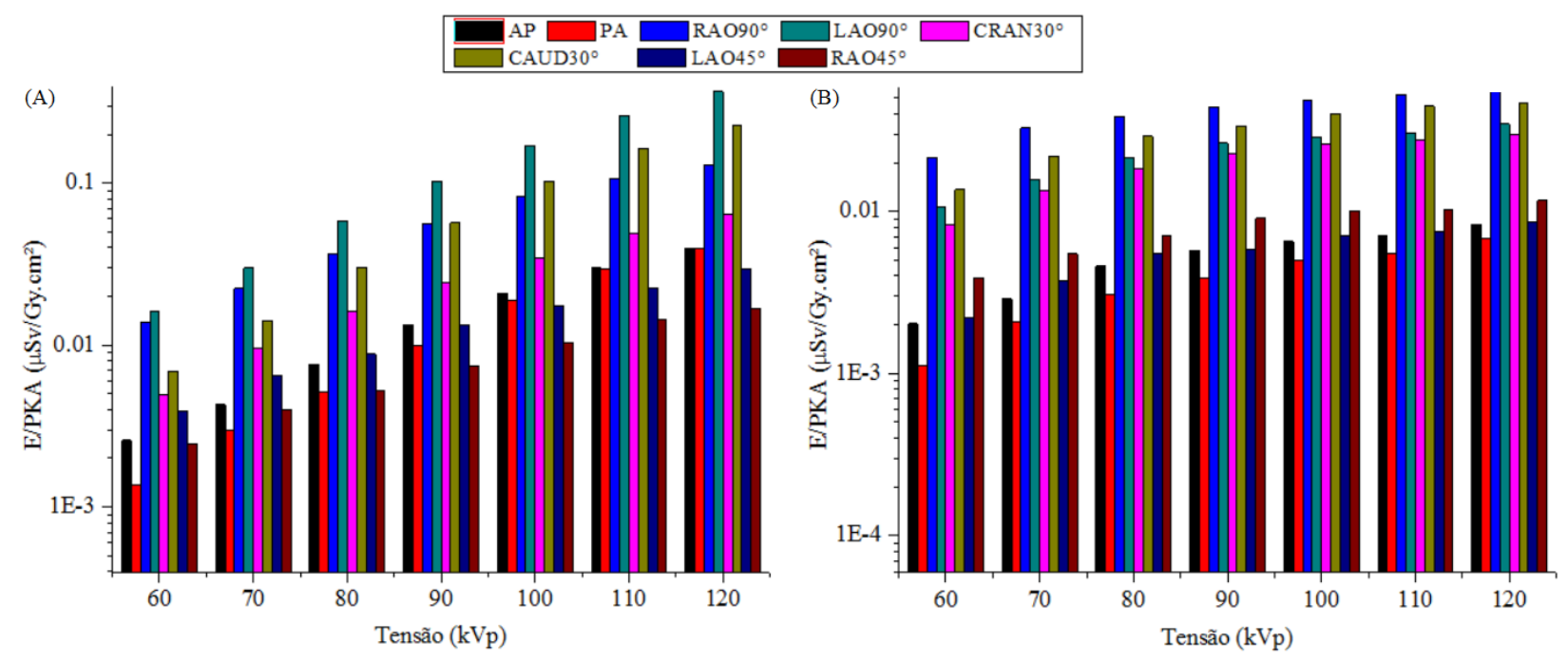

A radiação retroespalhada a partir da superfície de entrada do paciente é de alta intensidade. Assim, os valores de E/PKA serão sempre significativamente maiores quando o cardiologista está perto do tubo de raios $\mathrm{X}$, como na projeção $\mathrm{LAO90}{ }^{\circ}$, do que quando ele está ao lado do intensificador de imagem. Neste sentido, blindagens adicionais devem ser sempre adotadas.

O valor médio de E/PKA para o médico cardiologista foi de 4,7E-02 $\mu$ Sv/Gy.cm² e para a enfermeira foi de 1,8E-02 $\mu \mathrm{Sv} / \mathrm{Gy} \cdot \mathrm{cm}^{2}$, sendo a incerteza de $0,1 \%$ para os dois casos. Grandes variações de E/PKA foram observadas entre o médico cardiologista e a enfermeira. Estas 
diferenças são provavelmente devido às diferentes distâncias destes profissionais ao centro do campo de radiação. O médico cardiologista posiciona-se mais próximo ao paciente do que a enfermeira, que se localiza atrás do médico.

Grandes variações foram observadas entre os resultados das exposições ocupacionais deste estudo e os resultados experimentais relatados na literatura. Padovani e Rodella (2001) e Bor et al (2006) relataram resultados para médicos intervencionistas de unidade angiográfica. Nestes estudos, eles encontraram valores 0,230 e 0,132 $\mu$ Sv/Gy.cm², respectivamente. Estes resultados são superiores em 389, e 181\% relativos aos obtidos no presente estudo. Nos referidos estudos, não ficou claro a descrição dos equipamentos de proteção utilizados como cortina de chumbo junto a mesa cirúrgica, protetores suspensos e nem a espessura do avental plumbífero do médico. Os valores de E/PKA apresentados por estes estudos foram calculados utilizando algoritmos, que consistem apenas nas doses depositadas em dosímetros posicionados em regiões mais expostas do corpo, como tórax e pescoço, enquanto que neste estudo foram utilizados simuladores antropomórficos computacionais, em que cada voxel desempenha uma função de um dosímetro, de dimensão de poucos milímetros cúbicos, possibilitando obter de forma mais fiel os coeficientes de conversão para dose equivalente de cada órgão e tecidos e, consequentemente, uma estimativa mais precisa do CC para dose efetiva do que os apresentados nos estudos da literatura citados.

\section{CONCLUSÕES}

Os valores das razões E/PKA e DEP/PKA para o paciente concordam bem com os resultados experimentais apresentados na literatura. A diferença entre a média dos CCs calculados neste trabalho e os obtidos na literatura foi de $10 \%$ e $17 \%$ para CCs de dose efetiva e dose de entrada na pele do paciente, respectivamente, evidenciando que os resultados deste estudo possam ser uma ferramenta útil e confiável na avaliação dos efeitos estocásticos em procedimentos cardíacos de RI. A partir dos resultados, conclui-se também que os CCs calculados para todos os individuos crescem com o aumento da tensão no tubo. Devido à proximidade dos órgãos radiossensíveis do médico cardiologista ao centro espalhador de radiação (tórax do paciente), os 
maiores valores de E/PKA foram obtidos na projeção LAO90. Nesta projeção, os órgãos da região frontal, sobretudo aqueles localizados na altura do tórax e próximos da superfície e, consequentemente, do paciente, contribuíram para o aumento da razão E/PKA. Comparativamente com estudos utilizando simulador antropomórfico matemático, o resultado de E/PKA para o paciente deste estudo foi superior em 23\%, o que garante uma melhor estimativa no cálculo do risco para o paciente.

\section{AGRADECIMENTOS}

Os autores deste trabalho agradecem ao Prof. Dr. Richard Kramer, da Universidade Federal de Pernambuco, por ceder uma versão dos simuladores antropomórficos computacionais MASH e FASH. Os autores também agradecem às agências de fomento brasileiras: Fundação de Amparo à Pesquisa do Estado de São Paulo (FAPESP, Projetos No. 2013/15669-3 e 2013/21741-9), CAPES (Projeto Pró-Estratégia 1999/2012), CNPq (Projeto Universal 470753/2013-7), MCT: Projeto INCT Metrologia das Radiações em Medicina e Fundação de Amparo à Pesquisa do Estado de Minas Gerais (FAPEMIG, Project No. APQ-03049-15).

\section{REFERÊNCIAS}

BETSOU, S.; EFSTATHOPOULOS, E.P.; KATRITSIS, D.; FAULKNER, K..; PANAYIOTAKIS, G., Patient radiation doses during cardiac catheterization procedures, The British Journal of Radiology, vol. 71(846), p 634-639, 1998.

BOR, D.; SANCAK, T.; OLGAR, T.; ELCIM, Y.; ADANALI, A.; SANLIDILEK, U.; AKYAR, S., Comparasion of effective doses obtained from dose área product and air kerma measurements in interventional radiology, The British Journal of Radiology, vol. 77, p 315322, 2004. 
BOR, D.; ONAL, E.; OLGAR, T.; CAGLAN, A.; TOKLU, T. Measurement and estimation of cardiologist dose received in interventional examinations. AAPM 48th Annual Meeting (Orange County Convention Center Orlando, FL, USA, July 30-August 3), 2006.

BROADHEAD, D.A.; CHAPPLE, C.L.; FALKNER, K.; DAVIES, M.L.; MCCALLIUM, H., The impact of cardiology on the collective effective dose in the North of England, The British Journal of Radiology, vol. 70(833), p 492-497, 1997.

CASSOLA, V.F.; LIMA, V.J.; KRAMER, R.; KHOURY, H.J., FASH and MASH: Female and male adult human phantoms based on polygon mesh surfaces. Part II. Dosimetric calculations, Physics in Medicine and Biology, vol. 55(1), p 163-189, 2010.

CHIDA, K.; SAITO, H.; OTANI, H.; KOHZUKI, M.; TAKAHASHI, S.; YAMADA, S.; SHIRATO, K.; ZUGUCHI, M., Relationship between fluoroscopic time, dose-area product, body weight and maximum radiation skin dose in cardiac interventional procedures, American Journal of Roentgenology, vol. 186(3), p 774-778, 2006.

CRANLEY, K.; GILMORE, B.J.; FOGARTY, G.W.A; DESPONDS, L., Catalogue of diagnostic x-ray spectra and other data. Institute of Physics and Engineering in Medicine Report 78, York: IPEM, 1997.

EFSTATHOPOULOS, E.P.; MAKRYGIANNIS, S.S.; KOTTOU, S.; KARVOUNI, E.; GIAZITZOGLOU, E.; KOROVESIS, S.; TZANALARIDOU, E.; RAPTOU, P.D.; KATRITSIS, D.G., Medical personnel and patient dosimetry during coronary angiography and intervention, Physics in Medicine and Biology, vol. 48(18), p 3059-3068, 2003.

EC. EUROPEAN COMMISSION. European guidance on estimating population doses from medical $x$ - ray procedures, radiation protection 154, European commission, Luxembourg, 2008.

FAULKNER, K., Dose audit and optimization for radiological and interventional procedures, Journal of Medical Physics, vol. 33(1), p 35, 2008.

FAULKNER, K.; VANÓ, E., Deterministic effects in interventional radiology, Radiation Protection Dosimetry, vol. 94(1-2), p 95-98, 2001. 
ICRP 120. INTERNATIONAL COMMISSION ON RADIOLOGICAL PROTECTION, Radiological Protection in Cardiology, ICRP Publication 120, Ann. ICRP 42, 2013.

ICRP, 103. INTERNATIONAL COMMISSION ON RADIOLOGICAL PROTECTION, The 2007 Recommendations of the International Commission on Radiological Protection, ICRP Publication 103, Ann. ICRP 37 (2-4), 2007.

KARAMBATSAKIDOU, A.; TORNVALL, P.; SALEH, N.; CHOULIARAS, T.; LOFBERG, P.O.; FRANSSON, A., Skin dose alarm levels in cardiac angiography procedures: is a single DAP value sufficient?, The British Journal of Radiology, vol. 78(933), p 803-809, 2005.

KATRITSIS, D.; EFSTATHOPOULOS, E.; BETSTOU, S.; KOROVESIS, S.; FAULKNER, K.; PANAYIOTAKIS, G.; WEBB-PELOE, M.M., Radiation exposure of patients and coronary arteries in the stent era: A prospective study, Catheterization and Cardiovascular Interventions, vol. 51(3), p 259-264, 2000.

KHODADADEGAN, Y.; ZHANG, M.; PAVLICEK, W.; PADEN, R.G.; CHONG, B.; SCHUELER, B.A.; FETTERLY, K.A.; LANGER, S.G.; WU, T., Automatic monitoring of localized skin dose with fluoroscopic and interventional procedures, Journal of Digital Imaging, vol. 24(4), p 626-639, 2011.

LEUNG, K.; MARTIN, C.J., Effective dose for coronary angiography, The British Journal of Radiology, vol. 69(821), p 426-431, 1996.

MCPARLAND, B.J., A study of patient radiation doses in interventional radiological procedures, The British Journal of Radiology, vol. 71(842), p 175-185, 1998.

MILLER, D.L., Overview of contemporary interventional fluoroscopy procedures, Health Physics, vol. 95(5), p 638-644, 2008.

NCRP. Ionizing radiation exposure of the population of the United States. NCRP report 160. The National Council on Radiation Protection and Measurements, Bethesda, USA, 2009.

PADOVANI, R and RODELLA, C. A. Staff dosimetry in interventional cardiology. Radiation. Protection Dosimetry. vol. 94, p 99 -103, 2001. 
PARK, S. H; LEE, C.; LEE, J. K. Organ absorbed doses and effective doses to the patient and the medical staff in interventional radiology calculated from voxel phantom, Journal of Nuclear. Science and Technology, p 309 - 312, 2008.

PELOWITZ, D.B., MCNPX User's Manual (version 2.7.0). Report LA-CP-11-00438, Los Alamos National Laboratory, USA, 2011.

QUAI, E.; PADOVANI, R.; PETERZOL, A.; VANO, E.; GUIBELALDE, E.; TOIVONEN, M., Maximum skin dose assessment in interventional cardiology: Results in three different european hospitals, In: Proceedings of European Congress of Radiology, Vienna, 13, p 542, 2003.

SANTOS, W.S.; CARVALHO JR, A.B.; HUNT, J.G.; MAIA, A.F., Using the Monte Carlo technique to calculate dose conversion coefficients for medical professionals in interventional radiology, Radiation Physics and Chemistry, vol. 95, p 177-180, 2014.

SANTOS, W.S.; NEVES, L. P.; PERINI, A. P.; BELINATO, W.; CALDAS, L. V. E.; CARVALHO JR, A. B.; MAIA, A. F. Exposures in interventional radiology using Monte Carlo simulation coupled with virtual anthropomorphic phantoms, Physica Medica, vol. 31, p 929 - 933, 2015.

STRUELENS, L.; VANHAVERE, F.; SMANS, K. Optimisation de la dose patient pour applications radiologiques spécifiques, vol.42, p 551 -563, 2007.

TAPIOVARA, M.; LAKKISTO, M.; SERVOMAA, A., PCXMC-A PC-based Monte Carlo program for calculating patient doses in medical x-ray examinations, Helsinki, Finland: Finnish Centre for Radiation and Nuclear Safety (STUK), 1997.

WAGNER, L.K.; ARCHER, B.R.; COHEN, J., Management of patient skin dose in fluoroscopically guided interventional procedures, Journal of Vascular and Interventional Radiology, vol. 11(1), p 25-33, 2000. 